Journal of Mathematics and Statistics Studies

ISSN: 2709-4200

DOI: $10.32996 / j m s s$

Journal Homepage: www.al-kindipublisher.com/index.php/jmss

\title{
Global Structure of Determining Matrices for a Class of Differential Control Systems
}

\author{
Chukwunenye Ukwu' 8 (D) and Onyekachukwu Henry lkeh $^{2}$ \& (D) \\ ${ }^{1}$ Department of Mathematics, University of Jos, Nigeria \\ ${ }^{2}$ Department of Basic Sciences, Federal School of Medical Laboratory Technology (Science), Jos, Nigeria \\ $\square$ Corresponding Author: Onyekachukwu Henry Ikeh, E-mail: ikehhenry245@gmail.com
}

\section{ARTICLE INFORMATION ABSTRACT}

Received: April 08, 2021

Accepted: June 05, 2021

Volume: 2

Issue: 1

DOI: $10.32996 / j m s s .2021 .2 .1 .8$

\section{KEYWORDS}

Determining, controllability, generic, double time-delay, control system, Induction,

SumProdPerm, Summation infeasibility, Matrices, Greatest and least integer functions
This paper developed and established unprecedented global results on the structure of determining matrices of generic double time-delay linear autonomous functional differential control systems, with a view to obtaining the controllability matrix associated with the rank condition for the Euclidean controllability of the system. The computational process and implementation of the controllability matrix were demonstrated on the MATLAB platform to determine the controllability disposition of a small-problem instance. Finally, the work examined the computing complexity of the determining matrices.

\section{Introduction}

Literature is awash with research activities on the determining matrices of autonomous linear hereditary control systems, due to the fact that they constitute the most efficient mechanism and vehicle for the investigation of the Euclidean controllability of the above class of control systems, with considerable savings in computational time, using the rank condition on the corresponding controllability matrices, being the least computationally intensive when compared to indices of control systems matrices and controllability Grammians. Amongst the works of notable authors. Ukwu (2016) pioneered the introduction of the least integer function in the statement and proof of the necessary and sufficient conditions for the Euclidean controllability of linear hereditary systems of double time-delay: $\dot{x}(t)=A_{0} x(t)+A_{1} x(t-h)+A_{2} x(t-2 h)+B u(t) ; t \geq 0$ with an initial function: $x(t)=\phi(t), t[-2 h, 0], h>0$; this enhanced the computability of the controllability matrix in Gabasov and Kirillova (1976) and eliminated any ambiguity that could arise in its application. The proof in his work relied on the results in Ukwu (2014) and (Ukwu, 2016a), which also incorporated the characterization of Euclidean controllability in terms of the indices of control systems and appropriated Taylor's theorem as an indispensable tool. The article provided an illustrative example of the computation of the controllability matrices and stated the implication of Euclidean controllability.

(Ukwu, 2014) obtained the functional form of the determining matrices for the class of single-delay linear neutral autonomous control systems of the form:

$$
\frac{d}{d t}\left[x(t)-A_{-1} x(t-h)\right]=A_{0} x(t)+A_{1} x(t-h)+B u(t), t \geq 0 ; x(t)=\phi(t), t \in[-h, 0], h>0
$$

where $A_{-1}, A_{0}, A_{1}$ are $n \times n$ constant matrices with real entries, $B$ is an $n \times m$ constant matrix with real entries. The initial function $\phi$ is in $\left.C\left([-h, 0], \mathbf{R}^{n}\right)\right)$. The control $u$ is in the space $L_{\infty}\left(\left[0, t_{1}\right], \mathbf{R}^{n}\right)$.

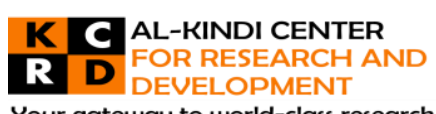

Your gateway to world-class research

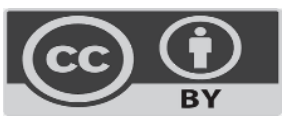

Published by Al-Kindi Center for Research and Development. Copyright (c) the author(s). This open access article is distributed under a Creative Commons Attribution (CC-BY) 4.0 license 
[6]: For $j, k \in \mathbf{N}, Q_{k}(j h)=\sum_{\left(v_{1} \ldots v_{j-k}\right) \in P_{-1(j), 0(k)}} A_{v_{1}} \ldots A_{v_{k}}$

$+\left[\sum_{\left(v_{1} \ldots v_{j}\right) \in P_{-1}(j-k), 1(k)} A_{v_{1}} \ldots A_{v j}+\sum_{r=1}^{k-1} \sum_{\left(v_{1}, \ldots v_{j+r}\right) \in P_{-1(r+j-k), 0(r), 1(k-r)}} A_{v_{1}} \ldots A_{v_{j+r}}\right] \operatorname{sgn}(\max \{0, j+1-k\})$ where

$P_{a\left(r_{a}\right), b\left(r_{b}\right), c\left(r_{c}\right)}$ denotes the set of all permutations of $\underbrace{a, a, \ldots, a}_{r_{a} \text { times }} \underbrace{b, b, \ldots, b}_{r_{b} \text { times }} \underbrace{c, c, \ldots, c}_{r_{c} \text { times }}$ : the permutations of the objects $a, b, c$ in

which $i$ appears $r_{i}$ times, $i \in\{a, b, c\}$.

Ukwu and Uche (2018) obtained restricted functional forms of determining matrices for a class of triple-delay linear control systems for certain pertinent parameters, thus bridging the knowledge gap in this area of acute research need by the exploitation of key facts about permutations, combinations of summation notations, change of variables techniques and the compositions of sigma and max functions. In the sequel, Ukwu and Uche (2018) established the result below on the functional form of the determining matrices for the class of triple-delay linear control systems:

$\dot{x}(t)=A_{0} x(t)+A_{1} x(t-h)+A_{2} x(t-2 h)+A_{3} x(t-3 h)+B u(t), t \geq 0 ; x(t)=\phi(t), t \in[-3 h, 0]$

where $A_{0}, A_{1}, A_{2}$ and $A_{3}$ are $n \times n$ constant matrices with real entries, $B$ is an $n \times m$ constant matrix with real entries. The initial function $\phi$ is in $C\left([-3 h, 0], \mathbf{R}^{n}\right)$, with sup norm. The control $u$, is in the space, $L_{\infty}\left(\left[0, t_{1}\right], \mathbf{R}^{n}\right)$.

Theorem on $Q_{k}(j h) ; 1 \leq k \leq j$ (Ukwu \& Uche, 2018):

For $1 \leq k \leq 5$

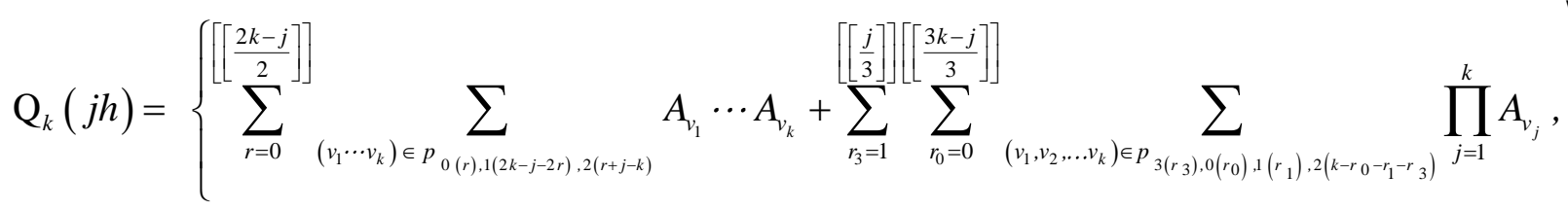
where

$r_{1}=\max \left\{0,3 k-2 j+3\left(r_{3}-r_{0}\right)\right\}$

Theorem on $Q_{k}(j h) ; 1 \leq k \leq j$ (Ukwu \& Uche, 2018):

For $1 \leq j \leq k ; j, k$ integers and $k \neq 0$

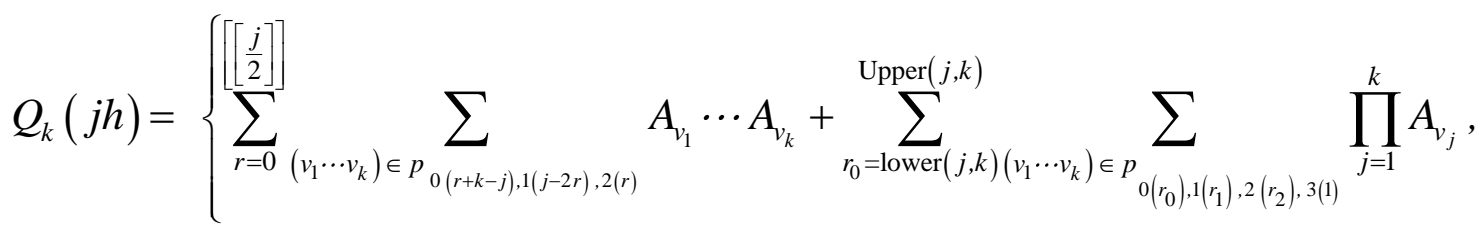

where

$$
\begin{aligned}
& \operatorname{lower}(j, k)=\left\{\begin{array}{l}
k-1, \text { if } j=3 \\
2+\operatorname{sgn}(\max \{0, k-j\}), j \geq 4
\end{array}\right. \\
& \operatorname{Upper}(j, k)=k-1-\operatorname{sgn}(\max \{0, j-3\})
\end{aligned}
$$




$$
r_{1}=k-\left(r_{0}+r_{2}+r_{3}\right), r_{2}=\left\{\begin{aligned}
\max \left\{\begin{array}{l}
k-j-1+3 r_{3}-r_{0}-\operatorname{sgn}(\max \{0, j-3\}) \\
+3 \operatorname{sgn}\left(\max \left\{0, r_{0}-2-\left[\left[\frac{6-j}{2}\right]\right]\right\}\right)
\end{array}\right\}, j \neq 3 \\
0 \quad \text { if } j=3
\end{aligned}\right\}
$$

However, these works were all limited to single time-delay control systems and double time-delay functional differential control systems of non-generic delays in the state variable. In an effort to extend the research activity the class of generic double time-delay control systems, (Ikeh \& Uche, 2021), amongst other results proved that the determining matrices of double time-delay linear autonomous functional differential control systems vanish if one of the time-delays is not an integral multiple of the other. The article went further to establish various functional forms and patterns of the determining matrices, $Q_{k}\left(j h_{1}\right)$, for $j \in\{0,1, \cdots, 7\}, k \in\{0,1, \cdots\}, h_{1}>0$, associated with the generic double time-delay linear autonomous control system:

$$
\begin{aligned}
& \dot{x}(t)=A_{0} x(t)+A_{1} x\left(t-h_{1}\right)+A_{2} x\left(t-h_{2}\right)+B u(t) ; t \geq 0 \\
& x(t)=\phi(t), t \in[-h, 0], 0<h_{1}, 0<h_{2}, h_{1} \neq h_{2}, h=\max \left\{h_{1}, h_{2}\right\} .
\end{aligned}
$$

where $A_{0}, A_{1}, A_{2}$ are $n \times n$ constant matrices with real entries $B$ is an $n \times m$ constant matrix with real entries. The initial function $\phi$ is in $C\left([-h, 0], \mathbf{R}^{n}\right)$, the space of continuous functions from $[-h, 0]$ into the real n-dimensional Euclidean space, $\mathbf{R}^{n}$ with norm defined by $\|\phi\|=\sup _{t \in[-h, 0]}|\phi(t)|$ (the sup norm). The control $u$ is in the space $L_{\infty}\left(\left[0, t_{1}\right], \mathbf{R}^{n}\right)$. The determining matrices for system (1.1) are the $n \times n$ matrix functions, $Q_{k}(s)$, which satisfy the system of equations:

$$
Q_{k}(s)=A_{0} Q_{k-1}(s)+A_{1} Q_{k-1}\left(s-h_{1}\right)+A_{2} Q_{k-1}\left(s-h_{2}\right)
$$

for $k=1,2, \ldots ; s>0$, with initial conditions:

$$
Q_{0}(0)=I_{n}, \quad Q_{0}(s)=0 ; s \neq 0
$$

These initial conditions guarantee the unique solvability of (1.1) (Gabasov \& Kirillova, 1976; Ikeh \& Ukwu, 2021). For notational simplicity, and without any loss of generality, let $h_{2}>h_{1}$.

This article adds to the body of knowledge by obtaining the structure of the afore-mentioned determining matrices for all pertinent global parameters and examining their computing complexity, thus filling the yawning gaps in Ikeh and Ukwu (2021).

\section{Main Result of This Work}

The main result of this research is given in the theorem below:

\subsection{Theorem on the Structure of the Determining Matrices, $Q_{k}\left(j h_{1}\right) ; j, k \in Z$}

Let $r_{0}, r_{1}, r_{2}$ be nonnegative integers and let $P_{0\left(r_{0}\right), 1\left(r_{1}\right), 2\left(r_{2}\right)}$ denote the set of all permutations of $\underbrace{0,0, \ldots, 0}_{r_{0} \text { times }} \underbrace{1,1, \ldots, 1}_{r_{1} \text { times }} \underbrace{2,2, \ldots, 2}_{r_{2} \text { times }}$ :

the permutations of the objects 0,1 and 2 in which $i$ appears $r_{i}$ times, $i \in\{0,1,2\}$. Let $[[]$.$] and [[[]]$.$] denote the greatest$ and least integer functions respectively. For $s \in \mathbf{R}$, let $Q_{k}(s)$ be the determining matrix of the system (1.1) with (1.2), under the standing hypotheses, subject to the initial condition: $Q_{0}(0)=I_{n}, Q_{0}(s)=0 ; s \neq 0$.

Then, for $j, k$ integers, $m=\{2,3,4, \ldots\}, q=m-1, h_{2}=m h_{1}$, 


$$
Q_{k}\left(j h_{1}\right)=\left\{\begin{array}{l}
{\left[\left[\frac{j}{m}\right]\right]} \\
\sum_{r=0}^{\left[\left[\frac{j}{m}\right]\right]} \sum_{\left(v_{1}, \ldots, v_{k}\right) \in P_{0}(q r+k-j), 1(j-m r), 2(r)} \prod_{i=1}^{k} A_{v_{i}}, \text { for } 0 \leq j \leq k, k \neq 0 \\
\sum_{\left.r=\left[\left[\left[\frac{j-k}{m-1}\right]\right]\right]\right]} \sum_{\left(v_{1}, \ldots, v_{k}\right) \in P_{0(q r+k-j), 1(j-m r), 2(r)}} \prod_{i=1}^{k} A_{v_{i}}, \text { for } 1 \leq k \leq j \\
I_{n}, \text { for } j=k=0 \\
0, \text { on the set }\{\operatorname{sgn}(\min \{j, k\})=-1\} \bigcup\{k=0, j \neq 0\} \bigcup\{0<k<j<m\} \bigcup\left\{h_{2} \neq m h_{1}\right\},
\end{array}\right.
$$

where the permutations in expressions (2.1) and (2.2) are all feasible.

\section{Proof:}

To prove (2.1), (2.2) and (2.4) for the first three sets under the union operation in (2.4) of the theorem, it is enough to prove that

$$
Q_{k}\left(j h_{1}\right)=\sum_{r=0}^{\left[\left[\frac{j}{m}\right]\right]} \sum_{\left(v_{1}, \ldots, v_{k}\right) \in P_{0(q r+k-j), 1(j-m), 2(r)}} \prod_{i=1}^{k} A_{v_{i}}
$$

and then weed out all summation infeasibilities by setting infeasible summations to zero, in accordance with mathematical convention. Assertion 1 of (Ikeh \& Ukwu, 2021) may then be invoked to conclude the proof of (2.4). Expression (2.3) is simply a restatement of the initial condition (1.4).

For notational optimality, expression (2.5) can also be written in the form:

$$
Q_{k}\left(j h_{1}\right)=\left[\sum_{r=0}^{\left[\left[\frac{j}{m}\right]\right]} \operatorname{SumProdPerm}\left[A_{0}(q r+k-j), A_{1}(j-m r), A_{2}(r)\right]\right.
$$

where, SumProdPerm $\left[A_{0}\left(r_{0}\right), A_{1}\left(r_{1}\right), A_{2}\left(r_{2}\right)\right]$ denotes the sum of the products of the permutations of $A_{0}, A_{1}$ and $A_{2}$ in which $A_{i}$ appears $r_{i}$ times, $i \in\{0,1,2\}$. We proceed first with the proof of (2.4) using (2.5):

If $j<0$, then $j-m r<0$ and $\left[\left[\frac{j}{m}\right]\right]<0$. Therefore $Q_{k}\left(j h_{1}\right)=0$, by the summation infeasibility of (2.5). If $k<0$ and $j>0$, then $[m-1] r+k-j<m\left[\left[\frac{j}{m}\right]\right]-j \leq 0$, thus $Q_{k}\left(j h_{1}\right)=0$, by permutation infeasibility. Both cases lead to the conclusion: $Q_{k}\left(j h_{1}\right)=0$ on the set $\{\operatorname{sgn}(\min \{j, k\})=-1\}$. If $k=0$ and $j>0$ then $[m-1] r+k-j<0$, thereby rendering all the permutations infeasible. Combine this with the case $j<0$, to conclude that $Q_{k}\left(j h_{1}\right)=0$ on $\{k=0, j \neq 0\}$. The case $0<k<j<m$ forces $\left[\left[\frac{j}{m}\right]\right]=0 \Rightarrow r=0 \Rightarrow(m-1) r+k-j=k-j<0$ ， resulting in permutation infeasibility; hence $Q_{k}\left(j h_{1}\right)=0$ on the set $\{0<k<j<m\}$. Lastly, the fact that $Q_{k}\left(j h_{1}\right)=0$ on the set $\left\{j>1, h_{2} \neq m h_{1}\right\}$ follows from (vii) of result (3.1) in (Ikeh \& Ukwu, 2021). This completes the proof of (2.4).

Plugging $j=0$ and $k \geq 1$, into (2.5) yields $Q_{k}(0)=\operatorname{SumProdPerm}\left[A_{0}(0), A_{1}(0), A_{2}(0)\right]=A_{1}^{k}$. This $\quad$ is consistent with the result from the determining equation: 


$$
\begin{aligned}
Q_{k}(0) & =A_{0} Q_{k-1}(0)+A_{1} Q_{k-1}\left(-h_{1}\right)+A_{2} Q_{k}\left(h_{1}-h_{2}\right) \\
& =A_{0} Q_{k-1}(0)=A_{0}^{2} Q_{k-2}(0)=\cdots=A_{0}^{k} Q_{0}(0)=A_{0}^{k}
\end{aligned}
$$

For the rest of the proof, the only remaining cases are constituted by the set $\min \{j, k\}=1$.

Consider $j=1, k \geq 1$ :

$j=1 \Rightarrow r=0$, in $(2.5) \Rightarrow Q_{k}\left(h_{1}\right)=\sum_{\left(v_{1}, \ldots, v_{k}\right) \in P_{0(0+k-1),(1-0), 2(0)}} \prod_{i=1}^{k} A_{v_{i}}=\sum_{\left(v_{1}, \ldots, v_{k}\right) \in P_{0(k-1),(1), 2(0)}} \prod_{i=1}^{k} A_{v_{i}}, h_{2}=m h_{1}$

$\Rightarrow Q_{k}\left(h_{1}\right)=\sum_{\left(v_{1}, \ldots, v_{k}\right) \in P_{(k-1) !(1)}} \prod_{i=1}^{k} A_{v_{i}}=\operatorname{SumProdPerm}\left[A_{0}(k-1), A_{1}(1)\right]$, for $h_{2}=m h_{1}, \forall k \geq 1$.

These two results correspond to (i) and (iv) of the established preliminary results (3.1) in (Ikeh \& Ukwu, 2021). Also,

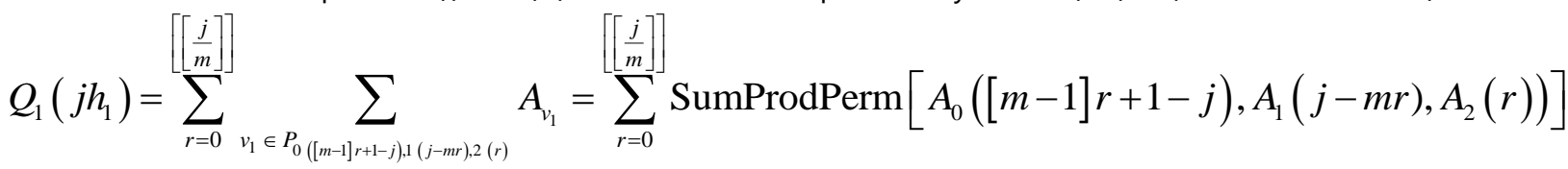

$j=0 \Rightarrow r=0 \Rightarrow Q_{1}\left(j h_{1}\right)=\sum_{v_{1} \in P_{0(1), 1(0), 2(0)}} A_{v_{1}}=A_{0} ; j=1 \Rightarrow r=0 \Rightarrow Q_{1}\left(j h_{1}\right)=A_{1}$;

$j=m \Rightarrow r \in\{0,1\}, r=0 \Rightarrow(m-1) r+1-j=1-m<0 \Rightarrow$ infeasible permutations,

$r=1 \Rightarrow(m-1) r+1-j=0, j-m r=0 \Rightarrow Q_{1}\left(j h_{1}\right)=A_{2} ; 2 \leq j<m \Rightarrow r=0 \Rightarrow(m-1) r+1-j<0$

$\Rightarrow$ infeasible permutations; $j>m \Rightarrow(m-1) r+1-j<(m-1) r+1-m$,

$r=0 \Rightarrow(m-1) r+1-m<1-m<0 \Rightarrow$ infeasible permutations; So $r \in\left[1,\left[\left[\frac{j}{m}\right]\right]\right]$

$\Rightarrow(m-1) r+1-j<m r-1+1-j<m\left[\left[\frac{j}{m}\right]\right]-j \leq j-j=0 \Rightarrow$ infeasible permutations.

Therefore $Q_{1}\left(j h_{1}\right)=0$, for $j \notin\{0,1, m\} \Rightarrow Q_{1}\left(j h_{1}\right)=\left\{\begin{array}{l}A_{j}, j \in\{0,1\} \\ A_{2}, j=m \\ 0, j \notin\{0,1, m\}\end{array}\right.$

From the determining equation:

$Q_{1}\left(j h_{1}\right)=A_{1} Q_{0}\left(j h_{1}\right)+A_{1} Q_{0}\left([j-1] h_{1}\right)+A_{2} Q_{0}\left([j-m] h_{1}\right)=\left\{\begin{array}{l}A_{j}, j \in\{0,1\} \\ A_{2}, j=m \\ 0, j \notin\{0,1, m\}\end{array}\right.$

Therefore the theorem is valid for $k=1$, for all $j \in\{1,2, \cdots\}$ prosecuted by the mathematical inductive principle.

Assume that the theorem is valid for all triple pairs, $k, Q_{k}\left(j h_{1}\right) ; j, k, Q_{k}\left(j h_{1}\right)$ for which $j+k \leq j+k$, for some $j, k: j \geq 2$ and $k \geq 2$. Then

$$
Q_{k+1}\left(j h_{1}\right)=A_{0} Q_{k}\left(j h_{1}\right)+A_{1} Q_{k}\left([j-1] h_{1}\right)+A_{2} Q_{k}\left([j-m] h_{1}\right)
$$

Now, $j-m \leq j-2<j-1<j$, for $m \in\{2,3, \cdots\} ; j<m \Rightarrow j-m<0 \Rightarrow Q_{k}\left([j-m] h_{1}\right)=0$.

We apply the induction hypothesis to the right hand side of $Q_{k+1}\left(j h_{1}\right)$ to get: 


$$
\begin{aligned}
& Q_{k+1}\left(j h_{1}\right)=A_{0} \sum_{r=0}^{\left[\left[\frac{j}{m}\right]\right]} \sum_{\left(v_{1}, \ldots, v_{k}\right) \in P_{O(q r+k-j), 1(j-m r), 2(r)}} \prod_{i=1}^{k} A_{v_{i}}+A_{1} \sum_{r=0}^{\left[\left[\frac{j-1}{m}\right]\right]} \sum_{\left(v_{1}, \ldots, v_{k}\right) \in P_{O(q r+k-[j-1]),(j-1-m r), 2(r)}} \prod_{i=1}^{k} A_{v_{i}} \\
& +A_{2} \sum_{r=0}^{\left[\left[\frac{j-m}{m}\right]\right]} \sum_{\left(v_{1}, \ldots, v_{k}\right) \in P_{0(q r+k-[j-m]),(j-m-m r), 2(r)}} \prod_{i=1}^{k} A_{v_{i}}
\end{aligned}
$$

Two cases arise: $j$ a multiple of $m$ and $j$ not a multiple of $m$

Case 1: $j$ a multiple of $m$; then: $\left[\left[\frac{j}{m}\right]\right]=\frac{j}{m},\left[\left[\frac{j-1}{m}\right]\right]=\frac{j}{m}-1=\left[\left[\frac{j-m}{m}\right]\right]$

The first component of the right hand side of (2.9) can be rewritten as:

$$
\sum_{r=0}^{\left.\left[\frac{j}{m}\right]\right]} \sum_{\left(v_{1}, \ldots, v_{k+1}\right) \in P_{0(q r+1 k+1]-j), 1}} \prod_{(j-m r), 2(r)}^{k+1} A_{v_{i}},\left(\text { with a leading } A_{0}\right)
$$

The second component can be rewritten in the form:

$$
A_{1} \sum_{r=o}^{\frac{j}{m}-1} \sum_{\left(v_{1}, \ldots, v_{k}\right) \in P_{O(q r+k-[j-1]),(j-1-m r), 2(r)}} \prod_{i=1}^{k} A_{v_{i}}
$$

We need to incorporate $\frac{j}{m}$ in the range of $r$ : If $r=\frac{j}{m}$; then $j-1-m r=j-1-j=-1$

Therefore the summation in (2.11) is infeasible; hence it is set equal to 0 .

Thus the case $r=\frac{j}{m}$ may be included in the expression (2.11), thus the second component can be rewritten as:

$A_{1} \sum_{r=o}^{\frac{j}{m}} \sum_{\left(v_{1}, \ldots, v_{k}\right) \in P_{(q r+\mid k+1\}-j),(j-1-m r), 2(r)}} \prod_{i=1}^{k} A_{v_{i}}=\sum_{r=0}^{\frac{j}{m}} \sum_{\left(v_{1}, \ldots, v_{k+1}\right) \in P_{0(q r+[k+1\}-j),(j-m r), 2(r)}} \prod_{i=1}^{k+1} A_{v_{i}} \quad$ (with a leading $\left.A_{1}\right)$

The third component of (2.9) may be rewritten in the form:

$$
A_{2} \sum_{r=0}^{\frac{j}{m}-1} \sum_{\left(v_{1}, \ldots, v_{k}\right) \in P_{0(q[r+1]+[k+1]-j), 1}} \prod_{(j-m(r+1)), 2(r)}^{k} A_{v_{i}}
$$

(2.13) is equivalent to the expression:

$$
A_{2} \sum_{r=0}^{\left[\left[\frac{j}{m}\right]\right]} \sum_{\left(v_{1}, \ldots, v_{k}\right) \in P_{0(q[r+1]+[k+1]-j), 1}} \prod_{(j-m(r+1)), 2(r)}^{k} A_{i=1}
$$

Using the change of variables converts (2.14) to the equivalent expression:

$$
A_{2} \sum_{r=1}^{\left[\left[\frac{j}{m}\right]\right]} \sum_{\left(v_{1}, \ldots, v_{k+1}\right) \in P_{0(q r+[k+1]-j), 1}} \prod_{(j-m r), 2(r-1)}^{k+1} A_{i=1}
$$


Expression (2.15) component for $r=0$ is infeasible and so equated to zero for that component.

Therefore (2.15), incorporating $r=0$ into (2.15) yields the equivalent form:

$A_{2} \sum_{r=0}^{\left.\left[\frac{j}{m}\right]\right]} \sum_{\left(v_{1}, \ldots, v_{k+1}\right) \in P_{0(q r+(k+1)-j), 1}} \prod_{(j-m r), 2(r-1)}^{k+1} A_{v_{i}}$

Expression (2.16) is equivalent to :

$$
\sum_{r=0}^{\left[\left[\frac{j}{m}\right]\right]} \sum_{\left(v_{1}, \ldots, v_{k+1}\right) \in P_{0(q r+\{k+1\}-j), 1}} \prod_{(j-m r), 2(r)}^{k+1} A_{v_{i}}\left(\text { with a leading } A_{2}\right)
$$

Combining expressions (2.9), (2.10), (2.12), and (2.17) we conclude that

$$
\begin{aligned}
& Q_{k+1}\left(j h_{1}\right)=\sum_{r=0}^{\left[\left[\frac{j}{m}\right]\right]} \sum_{\left(v_{1}, \ldots, v_{k+1}\right) \in P_{O(q r+k+1\}-j), 1(j-m r), 2(r)}} \prod_{i=1}^{k+1} A_{v_{i}}\left(\text { with a leading } A_{0}\right) \\
& \left.+\sum_{r=0}^{\frac{j}{m}} \sum_{\left(v_{1}, \ldots, v_{k+1}\right) \in P_{O(q r+\mid k+1-j),(j-m r), 2(r)}} \prod_{i=1}^{k+1} A_{v_{i}} \quad \text { (with a leading } A_{1}\right) \\
& +\sum_{r=0}^{\left[\frac{j}{m}\right]} \sum_{\left(v_{1}, \ldots, v_{k+1}\right) \in P_{0(q r+k+1-j), 1}} \prod_{(j-m r)), 2(r)}^{k+1} A_{v_{i}}\left(\text { with a leading } A_{2}\right) \\
& \text { Thus: } \quad Q_{k+1}\left(j h_{1}\right)=\sum_{r=0}^{\left[\left[\frac{j}{m}\right]\right]} \sum_{\left(v_{1}, \ldots, v_{k+1}\right) \in P_{O(q r+k+1]-j), 1(j-m r), 2(r)}} \prod_{i=1}^{k+1} A_{v_{i}} ; h_{2}=m h_{1}
\end{aligned}
$$

This concludes the proof of the theorem for case 1.

Case 2: $\quad j$ is not a multiple of $m$; then $\left[\left[\frac{j}{m}\right]\right]=\left[\left[\frac{j-\alpha}{m}\right]\right] ; \alpha \in\{1,2, \ldots, j-m\}$

Either $j<m$ or $j>m$. Subcase $1: j<m \Rightarrow Q_{k}\left([j-m] h_{1}\right)=0 ;\left[\left[\frac{j-\alpha}{m}\right]\right]=0, \alpha \in\{0,1, \cdots, j\}$ yielding the expression (2.21), as already established with the restriction $r=0$. Therefore,

$$
Q_{k+1}\left(j h_{1}\right)=\sum_{\left(v_{1}, \ldots, v_{k+1}\right) \in P_{0([k+1-j), 1(j), 2(0)}} \prod_{i=1}^{k+1} A_{v_{i}}
$$

proving the theorem for $j<m$. We now examine subcase 2: $j>m, j$ not a multiple of $m$.

Then $\left[\left[\frac{j}{m}\right]\right]=\left[\left[\frac{j-\alpha}{m}\right]\right]=\left[\left[\frac{j-m}{m}\right]\right]+1, \alpha \in\{1, \cdots, j-m\}$

Again the first component of (2.9) is the same as expression (2.10), the summation limits and permutation parameters being the same. Renumber it serially to get

$$
\sum_{r=0}^{\left.\left[\frac{j}{m}\right]\right]} \sum_{\left(v_{1}, \ldots, v_{k+1}\right) \in P_{(q r+[k+1\}-j), 1}(j-m r), 2(r)} \prod_{i=1}^{k+1} A_{v_{i}},\left(\text { with a leading } A_{0}\right)
$$

The second component of (2.9) is equivalent to: 
$A_{1} \sum_{r=0}^{\left.\left[\frac{j-1}{m}\right]\right]} \sum_{\left(v_{1}, \ldots, v_{k}\right) \in P_{0(q r+k-j j-1), 1(j-1-m r), 2(r)}} \prod_{i=1}^{k} A_{v_{i}}=\sum_{r=0}^{\left[\left[\frac{j}{m}\right]\right]} \sum_{\left(v_{1}, \ldots, v_{k+1}\right) \in P_{0(q r+\{k+1\}-j), 1(j-m r), 2(r)}} \prod_{i=1}^{k+1} A_{v_{i}}$ (with a leading $\left.A_{1}\right)(2.24) \quad$ The third component of (4.1) can be rewritten in the form:

$$
\begin{aligned}
& A_{2} \sum_{r=0}^{\left[\left[\frac{j-m}{m}\right]\right]} \sum_{\left(v_{1}, \ldots, v_{k}\right) \in P_{O(q r+k+11-j), 1(j-m(r+1)), 2(r)}} \prod_{i=1}^{k} A_{v_{i}}=A_{2} \sum_{r=0}^{\left[\left[\frac{j}{m}\right]\right]-1} \sum_{\left(v_{1}, \ldots, v_{k}\right) \in P_{0(q r+k k+1)-j), 1(j-m(r+1)), 2(r)}} \prod_{i=1}^{k} A_{v_{i}} \\
& =A_{2} \sum_{r=1}^{\left[\left[\frac{j}{m}\right]\right]} \sum_{\left(v_{1}, \ldots, v_{k}\right) \in P_{0(q r+[k+1]-j),(j-m r), 2(r-1)}} \prod_{i=1}^{k} A_{v_{i}} .
\end{aligned}
$$

If $r=0$, then $r-1=-1<0$. Therefore, the summations with $r=0$ vanish and the expression (2.24) above transforms to:

$$
\sum_{r=0}^{\left.\left[\frac{j}{m}\right]\right]} \sum_{\left(v_{1}, \ldots, v_{k+1}\right) \in P_{0(q r+[k+1\}-j), 1}} \prod_{(j-m r), 2(r)}^{k+1} A_{v_{i}}\left(\text { with a leading } A_{2}\right) \text {, }
$$

as already established in expression (2.17), since the limits and permutation parameters are the same. Combining (2.23), (2.24), and (2.26), the expression (2.9) translates to

$$
Q_{k+1}\left(j h_{1}\right)=\sum_{r=0}^{\left[\left[\frac{j}{m}\right]\right]} \sum_{\left(v_{1}, \ldots, v_{k+1}\right) \in P_{0(q r+[k+1]-j),(j-m r), 2(r)}} \prod_{i=1}^{k+1} A_{v_{i}} ; h_{2}=m h_{1}
$$

This concludes the proof for $j$ not a multiple of $m$. Hence the validity of the theorem expressed in the form of expression (2.5) established, with embedded infeasible summations.

To delete the infeasible summations, two cases arise: Case $1: j \leq k$; case $2: j \geq k$.

Case $1: j \leq k \Rightarrow[m-1] r+k-j \geq 0, j-m r \geq j-m\left[\left[\frac{j}{m}\right]\right] \geq j-j=0 \Rightarrow j-m r \geq 0$;

Case $2: j \geq k:[m-1] r+k-j \geq 0$ only if $r \geq \frac{j-k}{m-1}$. Since $r$ is a nonnegative integer, it

follows that $[m-1] r+k-j \geq 0$ only if $r \geq\left[\left[\left[\frac{j-k}{m-1}\right]\right]\right]$, guarateeing feasibility of all

permutations when $j \geq k$; needless to say that the permutations are all feasible for $j \leq k$.

\section{Therefore}




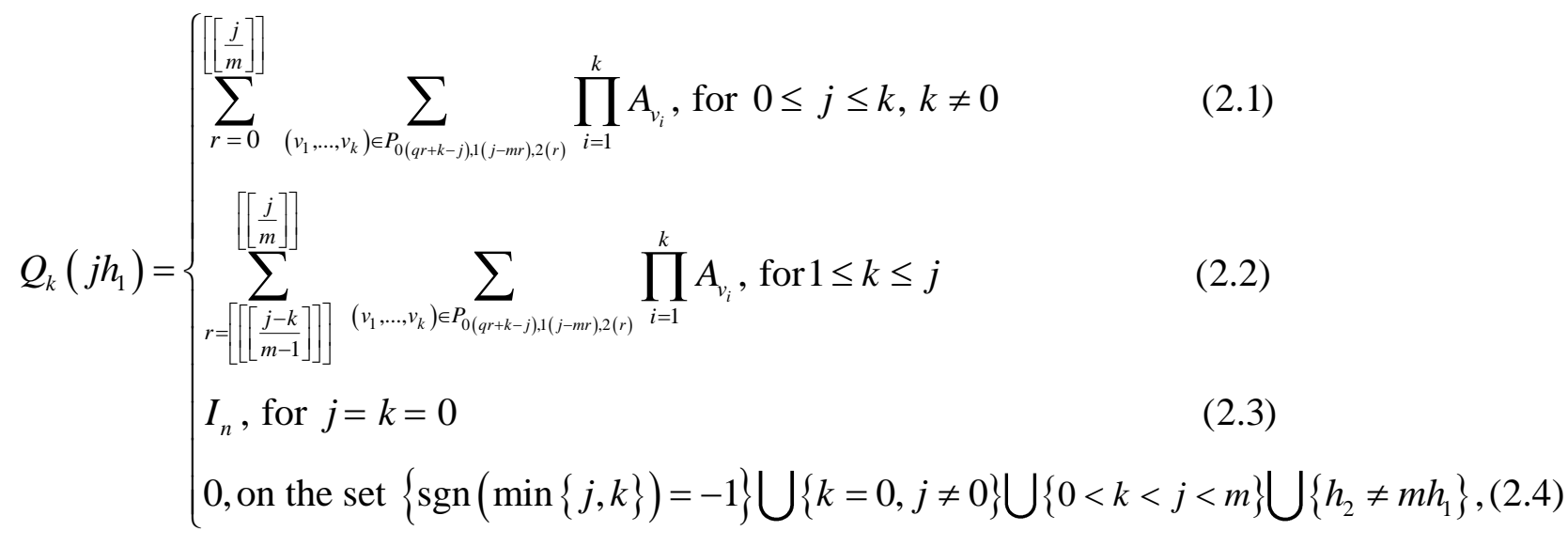

noting that expressions (2.3) and (2.4) have been established already. This completes the proof.

\section{Corollary}

Let $0 \leq j \leq k ; j, k$ integers, $k \neq 0$. Then for $h_{2}=m h_{1}, m \in\{j+1, j+2, \cdots\}$,

$Q_{k}\left(j h_{1}\right)=\sum_{\left(v_{1}, \ldots, v_{k}\right) \in P_{0(k-j), 1}(j),} \prod_{i=1}^{k} A_{v_{i}}=\operatorname{SumProdPerm}\left[A_{0}(k-j), A_{1}(j)\right]$

Proof: (i) is immediate from the fact that $\left[\left[\frac{j}{m}\right]\right]=0$, if $0 \leq j<m$.

\section{Analysis of the Degeneracy of the Determining Matrices for System (1.1)}

If $A_{2}=0$, in theorem (2.1), then the only surviving terms in the expression for $Q_{k}\left(j h_{1}\right)$ are the terms with $r=0$. It follows that:

$Q_{k}\left(j h_{1}\right)=\sum_{\left(v_{1}, \ldots, v_{k}\right) \in P_{0(k-j), 1(j)}} \prod_{i=1}^{k} A_{v_{i}} ; A_{2}=0, h_{2}=m h_{1}$

The functional forms in [2] can be established as follows:

For: $j>k \geq 1 ; p \in\{0,1, \ldots, k-1\} ; j, k, p$ integers

$$
Q_{k}\left(j h_{1}\right)=\sum_{\left(v_{1}, \ldots, v_{k}\right) \in P_{0(k-p-1),(p), 2(1)}} \prod_{i=1}^{k} A_{v_{i}} ; h_{2}=(j-p) h_{1}, j>2 p
$$

Observe here, that $A_{2}$ appears in every permutation in $Q_{k}\left(j h_{1}\right)$. Therefore, $Q_{k}\left(j h_{1}\right)=0$, if $j \geq k+1$ and $A_{2}=0$. Combining the above conditions, we deduce that the determining matrices for the free part of the system $\dot{x}(t)=A_{0} x(t)+A_{1} x\left(t-h_{1}\right)+B u(t)$ is given by:

$$
Q_{k}\left(j h_{1}\right)=\left\{\begin{array}{c}
\sum_{\left(v_{1}, \ldots, v_{k}\right) \in P_{0(k-j), 1(1)}} \prod_{i=1}^{k} A_{v_{i}} ; k \geq j \\
0 ; j \geq k+1
\end{array}\right.
$$

This result is consistent with the results for the same single-delay system in (Gabasov \& Kirillova, 1976), (Manitus \& Olbrot, 1976) and (Ukwu, 1992).

If $m=2$, then $q=1$; consequently theorem 2.1 degenerates to the following:

For $0 \leq j \leq k ; j, k$ integers, $k \neq 0, h_{2}=2 h_{1}$, 


$$
Q_{k}\left(j h_{1}\right)=\sum_{r=0}^{\left[\left[\frac{j}{2}\right]\right]} \sum_{\left(v_{1}, \ldots, v_{k}\right) \in P_{0(r+k-j), 1}} \prod_{i(j-2 r), 2(r)}^{k} A_{v_{i}} ; h_{2}=2 h_{1}
$$

This result is consistent with theorem (3.5.4) in (Ukwu, 2016).

\section{Theorem on Rank Conditions for the Euclidean Controllability of System (1.1)}

Let $[[[]]$.$] denote the least integer function (celling function) and let$

$$
\hat{Q}_{n}\left(t_{1}\right)=\left[Q_{0}(s) B, Q_{1}(s) B, \ldots, Q_{n-1}(s): s \in\left[0, t_{1}\right), s=0, h_{1}, \ldots,(n-1) h_{1}\right]
$$

where $Q_{k}(s)$ is a determining matrix for (1.1) defined by (1.3). Then system (1.1) is Euclidean controllable on $\left[0, t_{1}\right]$ if and only if $\operatorname{rank} \hat{Q}_{n}\left(t_{1}\right)=n$. Moreover $\hat{Q}_{n}\left(t_{1}\right)$ and $\operatorname{dim} \hat{Q}_{n}\left(t_{1}\right)$ are expressible as:

$$
\begin{aligned}
& \hat{Q}\left(t_{1}\right)=\left[Q_{k}(s) B: k \in\{0,1, \ldots, n-1\}, s \in\left\{0, h_{1}, \ldots,\left(\min \left\{(n-1),\left[\left[\frac{t_{1}-h_{1}}{h_{1}}\right]\right]\right]\right\}\right) h_{1}\right] \\
& \operatorname{Dim}\left[\hat{Q}_{n}\left(t_{1}\right)\right]=n \times \operatorname{mn}\left(\min \left\{\left[\left[\frac{t_{1}}{h_{1}}\right]\right]\right], n\right\}=n \times \operatorname{mn}\left(1+\min \left\{\left[\left[\left[\frac{t_{1}-h_{1}}{h_{1}}\right]\right]\right], n-1\right\}\right)
\end{aligned}
$$

This theorem is a modified version of that stated and proved in [7] to suit the system under consideration.

5.1 Illustrations of Non-Electronic Computations of $Q_{k}\left(j h_{1}\right)$

For $j, k \in\{3,4\}, j \neq k, m \in\{2,3,4\}$

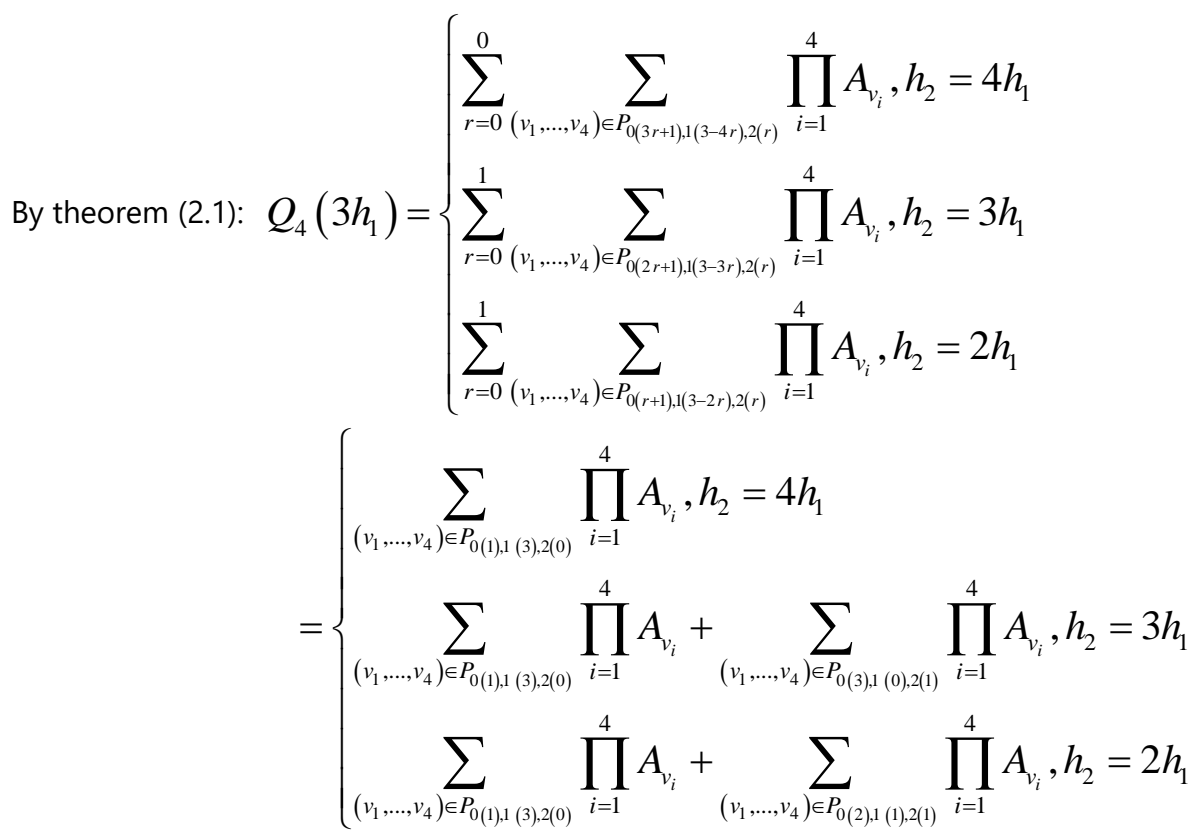

\footnotetext{
SumProdPerm $\left[A_{0}(1), A_{1}(3), A_{2}(0)\right], h_{2}=4 h_{1}$

$=\left\{\operatorname{SumProdPerm}\left[A_{0}(1), A_{1}(3), A_{2}(0)\right]+\operatorname{SumProdPerm}\left[A_{0}(3), A_{1}(0), A_{2}(1)\right], h_{2}=3 h_{1}\right.$

SumProdPerm $\left[A_{0}(1), A_{1}(3), A_{2}(0)\right]+\operatorname{SumProdPerm}\left[A_{0}(2), A_{1}(1), A_{2}(1)\right], h_{2}=2 h_{1}$
} 


$$
\begin{gathered}
=\left\{\begin{array}{l}
\text { SumProdPerm }\left[A_{0}(1), A_{1}(3)\right], h_{2}=4 h_{1} \\
\text { SumProdPerm }\left[A_{0}(1), A_{1}(3)\right]+\text { SumProdPerm }\left[A_{0}(3), A_{2}(1)\right], h_{2}=3 h_{1} \\
\text { SumProdPerm }\left[A_{0}(1), A_{1}(3)\right]+\text { SumProdPerm }\left[A_{0}(2), A_{1}(1), A_{2}(1)\right], h_{2}=2 h_{1}
\end{array}\right. \\
=\left\{\begin{array}{l}
A_{0} A_{1}^{3}+A_{1}^{2} A_{0} A_{1}+A_{1} A_{0} A_{1}^{2}+A_{1}^{3} A_{0}, h_{2}=4 h_{1} ; \\
A_{0} A_{1}^{3}+A_{1}^{2} A_{0} A_{1}+A_{1} A_{0} A_{1}^{2}+A_{1}^{3} A_{0}+A_{0}^{3} A_{2}+A_{2} A_{0}^{3}+A_{0}^{2} A_{2} A_{0}+A_{0} A_{2} A_{0}^{2}, h_{2}=3 h_{1} ; \\
A_{0} A_{1}^{3}+A_{1}^{2} A_{0} A_{1}+A_{1} A_{0} A_{1}^{2}+A_{1}^{3} A_{0}+A_{0} A_{1} A_{0} A_{2}+A_{0} A_{2} A_{0} A_{1}+A_{1} A_{2} A_{0}^{2}+A_{2} A_{1} A_{0}^{2} \\
+A_{0}^{2} A_{1} A_{2}+A_{0}^{2} A_{2} A_{1}+A_{0} A_{1} A_{2} A_{0}+A_{0} A_{2} A_{1} A_{0}+A_{1} A_{0}^{2} A_{2}+A_{2} A_{0}^{2} A_{1}+A_{1} A_{0} A_{2} A_{0}+A_{2} A_{0} A_{1} A_{0}, h_{2}=2 h_{1}
\end{array}\right. \\
\qquad \begin{array}{l}
\sum_{i=1}^{3} A_{v_{i}} ; h_{2}=(4-p) h_{1}, p \in\{0,1,2\} \\
Q_{3}\left(4 h_{1}\right)=\left\{\begin{array}{l}
\text { SumProdPerm }\left[A_{0}(2), A_{1}(0), A_{2}(1)\right], h_{2}=4 h_{1} \\
\text { SumProdPerm }\left[A_{0}(1), A_{1}(1), A_{2}(1)\right], h_{2}=3 h_{1} \\
\text { SumProdPerm }\left[A_{0}(0), A_{1}(2), A_{2}(1)\right], h_{2}=2 h_{1}
\end{array}\right.
\end{array} \\
\qquad \begin{array}{l}
A_{0}^{2} A_{2}+A_{2} A_{0}^{2}+A_{0} A_{2} A_{0}, h_{2}=4 h_{1} ; \\
A_{0} A_{1} A_{2}+A_{0} A_{2} A_{2}+A_{1} A_{0} A_{2}+A_{1} A_{2} A_{0}+A_{2} A_{1} A_{0}+A_{2} A_{0} A_{1}, h_{2}=3 h_{1} ; \\
A_{1}^{2} A_{2}+A_{2} A_{1}^{2}+A_{1} A_{2} A_{1}, h_{2}=2 h_{1}
\end{array}
\end{gathered}
$$

Clearly $Q_{3}\left(4 h_{1}\right) \neq Q_{4}\left(3 h_{1}\right)$ and in general: $Q_{k}\left(j h_{1}\right) \neq Q_{j}\left(k h_{1}\right)$, for $j \neq k$.

The computations are unwieldy and prohibitive for large parameter cases; a resort to electronic implementations becomes imperative.

By theorem 2.1; when $j=10, k=14$ and $m=7$, then

$$
Q_{14}\left(10 h_{1}\right)=\sum_{r=0}^{1} \operatorname{SumProdPerm}\left[A_{0}(6 r+14-10), A_{1}(10-7 r), A_{2}(r)\right] ; h_{2}=7 h_{1}
$$

$$
=\operatorname{SumProdPerm}\left[A_{0}(4), A_{1}(10), A_{2}(0)\right]+\operatorname{SumProdPerm}\left[A_{0}(10), A_{1}(3), A_{2}(1)\right] ; h_{2}=7 h_{1}
$$

The manual computations of this result are quite cumbersome. In general, there is an inherent computational intractability associated with these determining matrices. A resort to electronic implementation could be contemplated, but devising appropriate code for such an undertaking would task the ingenuity of even a professional computer programmer. However for small parameter instances, the implementation may be carried out on the MATLAB platform. The following section illustrates the implementation of determining matrices, controllability matrices and rank condition for Euclidean controllability for small parameter instances on the MATLAB platform.

\subsection{Electronic Implementation of Controllability Matrix and Rank Condition for System (1)}

This section is concerned with the prosecution of the above task for small problem instances on the MATLAB platform.

Let $A_{0}=\left(\begin{array}{lll}1 & 2 & 1 \\ 0 & 1 & 0 \\ 2 & 3 & 2\end{array}\right), A_{1}=\left(\begin{array}{ccc}1 & 1 & 1 \\ 1 & 2 & 3 \\ 1 & -1 & 1\end{array}\right), A_{2}=\left(\begin{array}{lll}1 & 1 & 1 \\ 1 & 1 & 2 \\ 0 & 1 & 2\end{array}\right), B=\left(\begin{array}{cc}1 & -1 \\ 2 & 1 \\ 1 & 2\end{array}\right), t_{1}=3, h_{1}=0.5, h_{2}=m h_{1}$

Then $m \in\{2,3, \ldots\}$, and $n=3$. Recall that the controllability matrix is 
$\hat{Q}_{n}\left(t_{1}\right)=\left[Q_{0}(s) B, Q_{1}(s) B, \ldots, Q_{n-1}(s): s \in\left\{0, h_{1}, \ldots, \min \left\{t_{1},(n-1) h_{1}\right\}\right\}\right]$, an

$n$ by $m n\left(1+\left[\left[\left[\min \left\{\frac{t_{1}}{h_{1}}, n-1\right\}\right]\right]\right]\right)$ concatenated matrix of $n\left(1+\left[\left[\left[\min \left\{\frac{t_{1}}{h_{1}}, n-1\right\}\right]\right]\right]\right)$ matrix objects, each of

dimension $n$ by $m$. Clearly,

$$
\begin{aligned}
& \hat{Q}_{3}(3)=\left[Q_{0}(s) B, Q_{1}(s) B, \ldots, Q_{2}(s): s \in\left\{0, h_{1}, 2 h_{1}\right\}\right] \\
& =\left[Q_{0}(0) B, Q_{1}(0) B, Q_{2}(0) B, Q_{0}\left(h_{1}\right) B, Q_{1}\left(h_{1}\right) B, Q_{2}\left(h_{1}\right) B, Q_{0}\left(2 h_{1}\right) B, Q_{1}\left(2 h_{1}\right) B, Q_{2}\left(2 h_{1}\right) B\right]
\end{aligned}
$$

The rank is invariant if the controllability matrix is pruned, with the deletion of associated zero matrices. Consequently,

$$
\begin{aligned}
\operatorname{rank} \hat{Q}_{3}(3) & =\operatorname{rank}\left[Q_{0}(0) B, Q_{1}(0) B, Q_{2}(0) B, Q_{1}\left(h_{1}\right) B, Q_{2}\left(h_{1}\right) B, Q_{1}\left(2 h_{1}\right) B, Q_{2}\left(2 h_{1}\right) B\right] \\
= & \operatorname{rank}\left[B, A_{0} B, A_{0}^{2} B, Q_{1}\left(h_{1}\right) B, Q_{2}\left(h_{1}\right) B, Q_{1}\left(2 h_{1}\right) B, Q_{2}\left(2 h_{1}\right) B\right]
\end{aligned}
$$

Below is the MATLAB code for obtaining the pruned $\hat{Q}_{3}(3)$ at $m=2,3,4, \ldots$ and its rank with the display of the result:

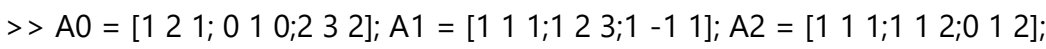

$>\mathrm{B}=[1-1 ; 21 ; 12] ; \mathrm{t} 1=3 ; \mathrm{n}=3 ; \mathrm{m}=2,3,4, \ldots ; \mathrm{h} 1=0.5$;

$>>\mathrm{Q} 00=$ eye (3); Q10 = A0; Q20 = A0^2; Q1h1 = A1; Q2h1 = A0*Q1h1+A1*Q10; Q12h1 = A2;

$>>\mathrm{Q} 22 \mathrm{~h} 1=\mathrm{A} 0 * \mathrm{Q} 12 \mathrm{~h} 1+\mathrm{A} 1{ }^{*} \mathrm{Q} 1 \mathrm{~h} 1+\mathrm{A} 2{ }^{*} \mathrm{Q} 10$;

$>>\mathrm{R}=$ horzcat $(\mathrm{Q} 00 * \mathrm{~B}, \mathrm{Q} 10 * \mathrm{~B}, \mathrm{Q} 20 * \mathrm{~B}, \mathrm{Q} 1 \mathrm{~h} 1 * \mathrm{~B}, \mathrm{Q} 12 \mathrm{~h} 1 * \mathrm{~B}, \mathrm{Q} 22 \mathrm{~h} 1 * \mathrm{~B})$;

$>>\operatorname{rank}(\mathrm{R})$

ans $=$

3

By the theorem 4, using the above parameters, the system (1.1) is Euclidean controllable for all values of $m$ on the interval [0,3].

\subsection{Computing Complexity of $Q_{k}\left(j h_{1}\right)$}

The computing complexity of an algorithm is a rough measure of its computational effort and is stated in terms of the size of the multiplications, the number of multiplications, additions, and comparisons involved in the computations of the relevant mathematical objects.

The computations of $Q_{k}\left(j h_{1}\right)$, even for moderately-sized $j$ and k could be quite tedious. An appeal to multinomial distributions yields the following measure of computing complexity with respect to multiplications and additions of the permutation objects in $Q_{k}\left(j h_{1}\right)$.

We define the cardinality of $Q_{k}\left(j h_{1}\right)$ as the number of nonzero components of $Q_{k}\left(j h_{1}\right)$ and it is 0 if $Q_{k}\left(j h_{1}\right)$ has all zero components. Denote the cardinality of $Q_{k}\left(j h_{1}\right)$ by $\left|Q_{k}\left(j h_{1}\right)\right|$. Then by the main theorem (2.1), the following is immediate:

$\left|Q_{k}\left(j h_{1}\right)\right|=0$, on the set $\{\operatorname{sgn}(\min \{j, k\})=-1\} \bigcup\{k=0, j \neq 0\} \bigcup\{0<k<j<m\} \bigcup\left\{h_{2} \neq m h_{1}\right\}_{\text {Note that for }}$ $\left|Q_{k}(0)\right|=1, k \geq 0$.

$0 \leq j \leq k, k \neq 0$, or for $j \geq k \geq 1, j, k$ integers, $\left|Q_{k}\left(j h_{1}\right)\right|$ is the number of nonzero product terms (component summand) in $Q_{k}\left(j h_{1}\right)$. 
Table 1: Computing Complexity Table For $Q_{k}\left(j h_{1}\right), k \neq 0$

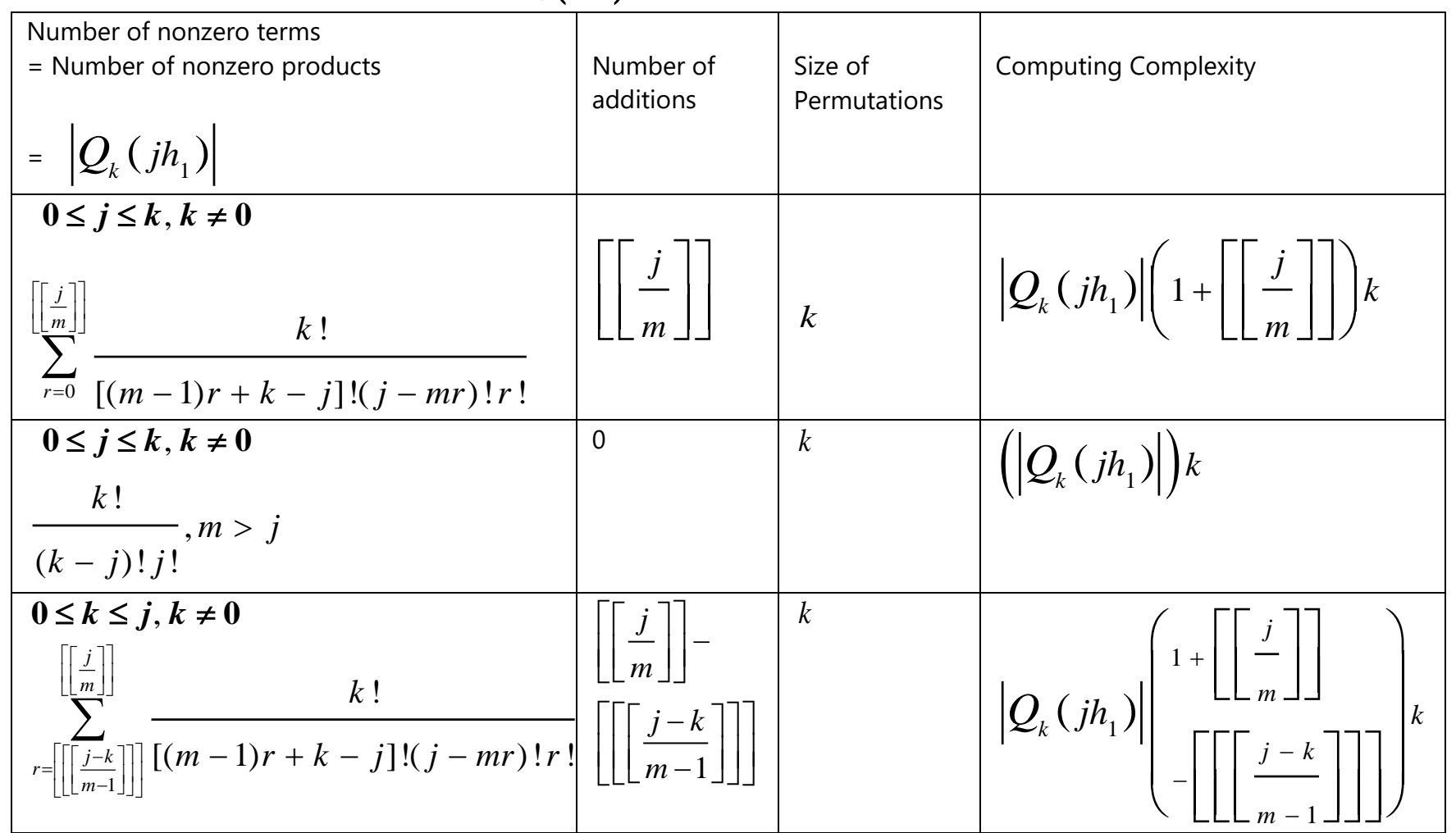

For $k<j<m,\left|Q_{k}\left(j h_{1}\right)\right|=0$. This follows from the fact that $Q_{k}\left(j h_{1}\right)=0$, if $k<j<m>0$. Note from the table that the cardinality and computing complexity of $Q_{k}\left(j h_{1}\right)$ is a decreasing function of $m$ and $\left|Q_{k}\left(j h_{1}\right)\right| \geq\left|Q_{j}\left(k h_{1}\right)\right|$ for $k, j>0$.

\section{Conclusion}

This article robustly and comprehensively established the structure of the determining matrices $Q_{k}\left(j h_{1}\right)$, of generic double timedelay linear autonomous functional differential control systems, (1.1), for arbitrary delay arguments $h_{1}$ and $h_{2}$, with illustrations of their electronic and non-electronic computations, for small problem instances. The article also performed an analysis of the degeneracy of the determining matrices. The work went further to provide the appropriate controllability matrices for the investigation of the Euclidean controllability of system (1.1) via rank condition determination. In the sequel the work examined the computational feasibility and computing complexity of the determining matrices, with a display of a table of the computing complexity. The results in this work have extended those in (Ukwu, 2016) and (Ikeh \& Ukwu, 2021) in leaps and bounds. Future research is the extension of these results to generic triple-delay autonomous linear control systems.

\section{References}

[1] Gabasov R. and Kirillova F. (1976). The Qualitative Theory of Optimal Processes, New York: Marcel Dekker Inc.

[2] Ikeh O. H. and Ukwu C. (2021). Fundamental Results on the Determining Matrices for a Certain Class of Hereditary Systems. Journal of Mathematics and Statistics Studies (JMSS).

[3] Manitus A. and Olbrot A. (1976). Finite Spectrum Assignment Problem for Systems with Delays, IEEE Trans. Autom. Control 24, $541-553$.

[4] Ukwu C. (1992). Euclidean Controllability and Cores of Euclidean Targets for Differential Difference Systems, North Carolina State University, Raleigh, USA: Unpublished Master of Science Thesis in Applied Mathematics with Operations Research.

[5] Ukwu C. (2014). The Structure of Determining Matrices for a Class of Double-Delay Control Systems, International Journal of Mathematics and Statistics Inventions (IJMSI), 2, 14-30.

[6] Ukwu C. (2014). The Structure of Determining Matrices for Single-Delay Autonomous Linear Neutral Control Systems, International Journal of Mathematics and Statistics Inventions (IJMSI), 2(3), 31-47.

[7] Ukwu C. (2016). Functional Differential Systems: Structures, Cores and Controllability, Deutschland: LAP LAMBERT Academic Publishing. 
[8] Ukwu C. (2016a), Necessary and Sufficient Conditions for Controllability of Double-Delay Autonomous Linear Control Systems, Journal of Scientific Research and Reports, 10, 1-9.

[9] Ukwu C. and Uche T. P. (2018). Parameter-Dependent Fundamental Forms of Determining Matrices for a Class of Triple-Delay Control Systems, International Research Journal of Natural and Applied Sciences, 5(10), 1-14. 